\title{
«On ne cherche pas des gens préparés à être ministre mais des gens qui sont prêts au changement »
}

Entretien avec Joséphine Ouédraogo, ancienne ministre de l'Essor familial et de la Solidarité nationale (1984-1987) lors de la présidence de Thomas Sankara

\section{Partie 1}

\author{
Hadrien Clouet et Maxime Quijoux
}

\begin{abstract}
Citer cet article : Clouet Hadrien et Quijoux Maxime (2021), " "On ne cherche pas des gens préparés à être ministre mais des gens qui sont prêts au changement". Entretien avec Joséphine Ouédraogo (Partie 1) », Revue d'Histoire Contemporaine de l'Afrique, en ligne. URL :
\end{abstract}

https://oap.unige.ch/journals/rhca/article/view/entclouetquijoux

Mise en ligne : 2 avril 2021

DOI : https://doi.org/10.51185/journals/rhca.2021.e476

Le 4 août 1983, un jeune militaire de 33 ans, Thomas Sankara, prend le pouvoir en HauteVolta, qu'il rebaptise Burkina Faso. À la tête d'une «coalition instable de groupuscules politiques et de factions militaires ${ }^{1}{ }^{1}$ il engage immédiatement une bifurcation politique d'ampleur, qui vise la souveraineté économique et démocratique. Cette Première Révolution (1983-1987) défend un projet de transformation sociale dont le socle repose sur le rejet de la soumission coloniale. Son gouvernement adopte une série de mesures phares : abrogation des privilèges de la fonction publique, recherche de l'autosuffisance alimentaire et manufacturière par la substitution aux importations et la réforme agraire, promotion des droits des femmes (lutte contre l'excision et la polygamie, formation professionnelle), vaccination, scolarisation de masse, protection de l'environnement, soutien aux mouvements de libération nationale dans le monde. Tout cela ne va pas sans oppositions : les cadres publics, désormais astreints à une discipline toute militaire; les chefs traditionnels, dessaisis de leur autorité coutumière; les catégories les plus favorisées, comme les militaires ou les professions syndicalisées, dont les revenus fondent; les syndicats, concurrencés par l'irruption des Comité de défense de la révolution dans la gestion des entreprises ${ }^{2}$ et l'opposition pro-soviétique ou social-démocrate.

Thomas Sankara est abattu le 15 octobre 1987 au cours d'un putsch mené par un de ses proches et membre du Conseil national de la révolution, Blaise Compaoré. Ce dernier demeure

\footnotetext{
1 Bianchini Pascal (2002), «Entre instrumentalisation et autonomisation. Journalistes et militants dans les luttes scolaires et universitaires au Sénégal et au Burkina Faso (années soixante-quatre-vingt-dix) ", Cahiers de la recherche sur l'éducation et les savoirs, 2002, 1, p. 165.

2 Phelan Craig (2016), «Plus ça change: Trade Unions, the Military and Politics in Burkina Faso, 1966 and 2014 », Labor History, 57(1), pp. 107-125.
} 
au pouvoir par la répression et la fraude électorale pendant 27 ans $^{3}$. Sa tentative de se représenter en 2014 conduit à la démission de plusieurs cadres de son parti et suscite des manifestations hostiles, notamment à l'initiative du mouvement civique "Le Balai citoyen". Lâché par l'armée, Blaise Compaoré fuit. Un Conseil national de désignation se réunit alors pour élire un président de la transition et un cabinet provisoire, en charge de réformer les institutions publiques et de préparer des élections libres en 2015. S'ouvre ainsi la Deuxième Révolution burkinabè.

Une femme relie ces deux séquences révolutionnaires: Joséphine Ouédraogo, sociologue ${ }^{4}$, ministre de I'Essor familial et de la Solidarité nationale sous Thomas Sankara (1984-1987), puis ministre de la Justice et Garde des Sceaux (2014-2015). Elle connaît intimement ces deux épisodes de l'histoire burkinabè, durant lesquelles elle a développé et mis en œuvre plusieurs politiques publiques. Entre 1984 et 1987, ses réformes familiales ont égalisé le statut des femmes et des hommes, pavant la voie du Code des personnes et de la famille, définitivement adopté en $1989^{5}$. En exil après l'assassinat de Sankara, elle œuvre au sein de la Commission économique pour l'Afrique et de I'ONG Enda-Tiers Monde, en faveur du développement humain et des droits des femmes africaines. Puis, elle est appelée au ministère de la Justice en 2014, au lendemain de l'insurrection populaire. Là, elle supervise la restructuration d'un système judiciaire après des décennies d'inféodation au régime de Blaise Compaoré.

Comment devient-on ministre d'un gouvernement révolutionnaire, qui plus est en tant que femme sociologue ? Comment des mesures de changement social sont-elles fabriquées, de leur conception et à leur exécution? Quelle place les sciences humaines et sociales, en particulier la sociologie, occupent-elles dans ce processus ? Ces questions s'inscrivent dans des trajectoires intellectuelles tournées vers les mobilisations et les initiatives gouvernementales de transformation sociale. Hadrien Clouet s'est investi dans des cadres internationaux de travail critique à l'égard des dettes publiques illégitimes, rencontrant des acteurs fortement marqués par le sankarisme ${ }^{6}$. Maxime Quijoux, quant à lui, s'intéresse depuis le début des années 2000 aux conflits du travail et à l'économie sociale et solidaire, dans ses dimensions syndicales et internationales Par ailleurs, en tant que sociologues, nous nous intéressons aux usages gouvernementaux de notre discipline et à ses potentialités de transformation. Le parcours de Joséphine Ouédraogo, découvert dans des écrits politiques et journalistiques, nous offre une occasion rare de pouvoir explorer simultanément ces enjeux.

Trois enseignements se dégagent de cet échange proposé en deux parties. D'abord, les propos de l'ancienne ministre de l'Essor familial rappellent le poids à la fois des réseaux d'interconnaissance et d'affinité dans le processus de formation d'un nouveau groupe de dirigeants. Cette sélection des cadres s'opère néanmoins dans le cadre du gouvernement par

\footnotetext{
3 Hagberg Sten, Kibora Ludovic, Ouattara Fatoumata et Konkobo Adjara (2015), " Au cœur de la révolution burkinabè », Anthropologie et développement, 42-43, pp. 199-216.

${ }^{4}$ Elle est l'autrice d'une série de publications, dont (1989) Rapport entre droit foncier traditionnel et droit moderne illustré par l'impact du régime Société sur le statut des femmes au Burkina Faso, Giuffré édition, ou (1996) «Les femmes chefs de ménage en zone rurale du Burkina Faso », in J. Bisillat, Femmes du Sud, chefs de famille, Paris, Karthala, pp. 99-107.

5 Dabo Aïssata (2017), L'égalité de l'homme et de la femme dans le mariage en Afrique noire francophone. Étude comparée des droits du Bénin, du Burkina Faso et du Mali, Thèse de doctorat en droit privé, Université de Bordeaux et Université d'Abomey-Calavi.

${ }^{6}$ Notamment lors de son activité dans le European Research Network on Social and Economic Policy ou le Sommet pour un Plan B.
} 
la prédominance de qualités morales, combinée à un souci d'une connaissance fine des problématiques populaires (« de terrain », dirait-on aujourd'hui), pour constituer un groupe de «technocrates» - terme étonnamment mobilisé par Sankara. Ce faisant, elle témoigne également des aspirations spécifiques d'une sociologue au pouvoir, soucieuse de repenser les rapports entre l'État et ses ressortissants et de développer de nouvelles approches de la démocratie, notamment auprès des groupes sociaux marginalisés (femmes, orphelins, vagabonds, prostituées).

Ensuite, ses propos rappellent l'importance des acquis révolutionnaires mais aussi les difficultés d'une telle politique. Dans ce contexte de changements rapides, les résistances sont multiples, à commencer par l'inertie bureaucratique et les habitudes associées à des conditions d'existence difficiles. Le régime de Sankara apparaît comme un cas paradigmatique, tant les ambitions gouvernementales contrastent avec les possibilités objectives du pays. En ce sens, la nomination d'une femme, qui plus est à la tête d'un ministère de "l'Essor familial », montre la détermination à transformer les rapports de genre. Les difficultés n'apparaissent pas moindres lorsqu'elle est rappelée en 2014 et nommée Garde des Sceaux : si les ressources du pays se sont accrues, elle fait désormais face à une administration constituée mais héritière du régime autoritaire.

Joséphine Ouédraogo témoigne enfin des mobilités internationales des élites politiques et des compétences acquises lors de sa formation de sociologue et de son expérience ministérielle. Inquiétée après l'assassinat de Thomas Sankara, elle parvient néanmoins à quitter le pays et, surtout, à convertir son capital d'expertise dans des bureaux d'études, des organismes gouvernementaux, des institutions onusiennes ou des ONG. Ainsi, elle exerce huit activités professionnelles différentes dans cinq pays, entre la fin de la Première Révolution en 1987 et le début de la Deuxième Révolution en 2014. La démarche empirique a constitué un fil rouge tout au long de sa carrière, nourrissant ses missions dans les différentes arènes nationales et internationales où elle est intervenue. À chaque demande correspond un besoin systématique de connaissance qui passe par la mise à distance des préjugés - y compris les plus vertueux et les plus révolutionnaires - par la mobilisation d'enquêtes statistiques ou d'entretiens auprès des populations qui permettent d'ajuster aux mieux les mesures sociales.

C'est pour nous exposer ces différentes perspectives qu'elle accepte notre sollicitation, le 23 septembre 2020 à 11 heures par visioconférence : elle depuis son bureau d'ambassadrice à Rome, nous depuis un bureau du Conservatoire national des arts et métiers. Au cours de la retranscription, nous l'avons sollicitée pour clarifier des noms, des dates et des lieux. Nous lui avons ensuite envoyé l'entretien retranscrit, qu'elle a relu, corrigé et validé. 


\section{La formation d'une sociologue burkinabè}

\section{Hadrien Clouet : Dans quel milieu familial avez-vous vu le jour ?}

J'ai vécu mon enfance à Koudougou, où je suis née en 1949. Mes parents étaient issus de familles d'agriculteurs, mon père a été scolarisé mais ma mère était illettrée. Mon père [Henri Guissou] était administrateur public, puis politicien voltaïque, parlementaire français, député des colonies d'Afrique occidentale française. Il voyageait beaucoup durant ma jeunesse. Un an après l'indépendance de la Haute-Volta en 1961, il a été nommé ambassadeur à Paris. Je suis donc brutalement passée d'élève d'une école primaire dans un quartier semi-urbain de Koudougou à une école privée tenue par une congrégation religieuse à Paris, comme fille d'ambassadeur.

Demi-pensionnaire, j'ai reçu une éducation mixte. Ma mère voulait préserver nos racines sociales et culturelles. En face, mon père, un homme public, nous a jetés profondément dans la modernité de l'époque. C'était une acrobatie psychoculturelle extraordinaire pour notre famille. Ma mère, épouse illettrée d'ambassadeur, a gardé son comportement et ses valeurs traditionnelles alors qu'elle a été plongée pendant 10 ans dans le milieu public diplomatique (Paris puis Bonn). Elle est restée elle-même, sans chercher à emprunter les apparences ni les comportements des autres épouses d'ambassadeurs, instruites et modernes; de nature très réservée, elle ne cherchait ni à briller ni à parler un français impeccable. Elle était dans sa peau de femme, épouse et mère, armée de sa culture paysanne. Bref, c'est dans ce cadre familial que j'ai passé mon bac, en 1968.

\section{Maxime Quijoux : Comment avez-vous vécu cette période à Paris ?}

J'avais 18 ans et avec mes camarades de classe nous étions plutôt témoins de ce qui se passait dans les rues. Je ne me suis pas rendue sur les barricades mais je me sentais concernée par les messages et les revendications des jeunes. Mes copines de classe et moi, nous étions sensibles aux discours et aux slogans sur la libération des femmes, la libération de la parole des jeunes, la libération de l'emprise familiale sur les jeunes, etc. Nous en parlions, mais sans faire de vagues dans notre école religieuse et très conservatrice. La révolution de Mai 68 a eu un impact sur notre baccalauréat, puisque toutes les épreuves furent passées à l'oral. En outre, cela brisait la distance habituelle entre les professeurs et les élèves, plutôt compliquée pour les plus timides comme moi.

À l'époque, après mon bac, j'ai été sollicitée par l'Association des étudiants voltaïques, un mouvement de gauche. Mais je trouvais leur idéologie trop tranchante par rapport à ce que je voyais de mon père, qui défendait corps et âme les intérêts de son pays, tout en conservant des liens avec son village où il nous ramenait chaque vacance. J'estimais qu'on pouvait appartenir à un milieu social aisé et privilégié, tout en étant attaché à ses racines sociales et sans être « ennemi du peuple».

En 1971, mes parents devaient rentrer en Haute-Volta, en raison de la retraite de mon père. J'avais néanmoins droit à une bourse d'études universitaires. J'ai opté pour l'Institut de travail social et de recherches sociales de Montrouge. Après trois ans, j'y ai obtenu mon diplôme d'État en service social. 


\section{Certains enseignants à Montrouge vous ont-ils marquée?}

Oui ! En sociologie on avait un professeur latino-américain dont j'oublie le nom. II nous a beaucoup sensibilisés sur les théories du changement social et notamment la lutte contre la pauvreté par l'éducation et la conscientisation des adultes. Il y avait aussi Madame Miské, la directrice de la section internationale de l'Institut. Une militante du développement par la participation populaire. Et puis il y avait le Professeur Gentilini, qui nous a enseignés la médecine tropicale. Ce sont les professeurs qui m’ont le plus marquée.

Ma formation à Montrouge m'a appris à aimer la sociologie, comme un instrument d'analyse des mutations sociales en lien avec les modèles de développement. Je découvrais la pauvreté comme la résultante d'un processus d'exclusion sociale, politique et culturelle, et non pas comme une situation de simple précarité matérielle. En plus, les étudiants de la section internationale devaient faire chaque année un stage dans leur pays, au sein d'une institution ou d'un projet de développement social. Cette formation m'a poussée à étudier la sociologie. En conséquence, une fois titulaire du diplôme d'État, je n'ai pas souhaité exercer le métier d'assistante sociale. Je me suis plutôt inscrite en sociologie à I'Université René Descartes, en vue d'avoir la licence. Je me considère comme une praticienne de la sociologie de développement, surtout grâce à mon premier travail dans un bureau d'études privé voltaïque, la SAED [Société africaine d'études de développement], qui m'a recrutée en 1974 comme chargée d'études sociologiques.

\section{Hadrien Clouet : En quoi consistait votre travail ?}

J'accompagnais les équipes d'ingénieurs, d'économistes et d'agronomes pour évaluer des projets sur le terrain: il s'agissait de projets de développement agricole (mécanisation, aménagements hydroagricoles, etc.), de projets de désenclavement, d'investissements socioéconomiques, d'éducation communautaire, de sensibilisation et de formation des femmes, etc. J'étais chargée d'analyser les composantes sociales ou sociologiques de ces projets. Par exemple, en évaluant les résultats économiques d'un programme de mécanisation agricole par I'accès au crédit, ma tâche consistait à analyser l'influence des structures sociales et des sphères de décisions au niveau familial sur l'utilisation des bénéfices issus de la production agricole. On passait des semaines sur le terrain, avec les paysans, les jeunes et les femmes.

Ainsi, je peux dire que je réconciliais la découverte des réalités sociales de mon pays avec ce que j'avais appris à Montrouge et à René Descartes. Je découvrais aussi comment fonctionnait le monde du développement, c'est à dire les institutions, les acteurs et les mécanismes par lesquels étaient introduites les innovations dans le monde rural. Je développais peu à peu un esprit critique sur ces schémas de développement qui en réalité proposaient des solutions toutes faites aux paysans, sans les associer aux choix des solutions ni aux méthodes utilisées pour les intégrer au processus de modernisation. J'écrivais quelques articles, j'accordais parfois des interviews et j'animais des ateliers dans le cadre de mon travail, sur des approches participatives et sur la condition des femmes rurales. J'ai exercé cette tâche de chargée d'études sociologiques pendant huit ans. 


\section{Maxime Quijoux : Comment viviez-vous la période politique de la fin des années $1970 ?$}

À l'époque, la marmite bouillonnait au Burkina Faso. Les syndicats, les élèves, les associations de la société civile étaient régulièrement ligués contre le régime en place depuis 14 ans, présidé par le général Sangoulé Lamizana (1966-1980). Il y a eu un premier coup d'État en 1980. Ensuite, un deuxième coup d'État deux ans plus tard. Et puis, pendant ce temps, les forces progressistes menées par Thomas Sankara, à travers des leaders d'associations de la société civile et de mouvements politiques, se préparaient pour susciter un changement radical en août 1983. Mais je ne faisais partie d'aucun de ces mouvements-là. J'avais été recrutée en 1982 comme sociologue à l'Autorité des aménagements des vallées des Volta, chargée d'un vaste programme de repeuplement de terres agricoles assainies de l'onchocercose ${ }^{7}$. Très vite, on $\mathrm{m}^{\prime} \mathrm{a}$ nommée directrice des études dans cette structure autonome placée sous la tutelle du ministère de l'Agriculture. Et c'est de là, brutalement, en août 1984, que le président Thomas Sankara m'appelle à rejoindre son gouvernement révolutionnaire.

\section{Gouverner dans l'urgence}

\section{Comment l'expliquez-vous, et comment se déroule ce moment ?}

J'ai été très surprise. Pas par le coup d'État de Thomas Sankara, parce qu'on sentait venir les choses. Mais il tenait des discours très tranchants contre l'impérialisme et le néocolonialisme et prenait des sanctions très sévères contre les réactionnaires et ceux qui étaient considérés comme des ennemis de la révolution. En plus, c'était un militaire politiquement engagé, c'était un patriote qui exprimait clairement et ouvertement sa révolte, son opposition à toutes les forces réactionnaires et les complices des pouvoirs impérialistes occidentaux en Afrique. Cette violence me faisait plutôt peur. Même si j'adhérais pleinement aux valeurs qu'il défendait, celles de justice sociale, d'indépendance politique, de liberté, de démocratie et de développement populaire, en tant que sociologue, j'étais convaincue qu'un changement politique et sociétal ne pouvait pas s'opérer avec brutalité, ni dans l'urgence.Nous nous connaissions et nous nous fréquentions parce que sa femme était une amie de ma sœur. Au téléphone, il m'a donné rendez-vous à son bureau et il m'a dit : «La révolution a besoin de toi ». Ils venaient de passer le cap de leur première année de gouvernance révolutionnaire au cours de laquelle ils ont annoncé les couleurs, sans ambiguïté ni compromission. Ils avaient pris des mesures très difficiles, qui avaient suscité des remous.

II m'explique: "J'ai besoin de gens comme toi, parce que je veux composer mon nouveau gouvernement avec des technocrates plutôt que des idéologues ». Je lui réponds que je ne suis pas du tout préparée à être ministre et que j'étais prête à travailler pour la révolution dans un poste de directeur technique ou de chef d'institution. II me répond : " On ne cherche pas des gens préparés à être ministre mais des gens qui sont prêts au changement. Tu connais le terrain, tu parcours la brousse, tu es en phase avec les paysans, tu travailles de manière

\footnotetext{
${ }^{7}$ L'onchocercose est une maladie occasionnée par un ver parasite qui cause notamment la cécité.
} 
concrète. Nous, nous voulons que la révolution se connecte aux réalités sociales. C'est cette dimension que je voudrais que tu apportes, avec d'autres aussi, que je vais appeler au gouvernement ».

Je me suis dit que c'était intéressant, je l'ai remercié pour sa considération et lui ai répondu que j'allais y réfléchir et consulter quelques proches. II approuve. Et ensuite, pendant que je discute avec mes proches, j'entends la radio qui annonce la composition du gouvernement, avec mon nom au poste de ministre de l'Essor familial et de la Solidarité nationale [Rires]. Après notre entretien, il a considéré que ça allait marcher. En septembre 1984, je suis donc nommée ministre. Sa méthode consistait à rencontrer chaque membre du gouvernement pour lui dire : «Voilà ce que je veux, voilà ma vision concernant le secteur qui vous est confié ». Dans mon cas, il m'a expliqué en quoi consistait la différence entre la politique sociale portée par l'ancien ministère des Affaires sociales, et sa vision d'une politique d'Essor familial et de Solidarité nationale. La politique sociale des régimes précédant la révolution se limitait essentiellement à des activités d'aide sociale aux femmes, à l'enfance, aux personnes vulnérables, et toute cette approche induisait et reproduisait un esprit d'assistanat social. C'était un héritage de la politique sociale de la France à l'époque.

Bref, il poursuit : « Je veux rompre avec tout ça. Je veux que ce ministère contribue à un changement de notre société. Pour nous aider à relever le statut de la femme, je vais créer I'Union des femmes burkinabè pour inciter les femmes à s'organiser dans un mouvement politique d'émancipation. C'est aux femmes de défendre leurs droits. Je créerai les conditions pour qu'elles puissent s'exprimer. Mais toi, crée les conditions institutionnelles et techniques, pour que le statut des femmes change sur le plan économique et juridique au sein de la famille. J'y ajoute l'aspect Solidarité nationale pour lutter contre l'exclusion, la pauvreté et l'extrême pauvreté, dont nous sommes les premiers responsables, car c'est notre système socioéconomique et notre mode de gouvernance qui engendrent les exclusions et l'appauvrissement d'une partie de la population. À présent nous voulons assumer notre responsabilité vis-à-vis des laissés pour compte... car nous ne voulons plus déléguer cette responsabilité à des ONG extérieures et à nos partenaires publics extérieurs pour nous apporter de l'assistance qui, en plus, ne résout pas le fond du problème. Donc, je te demande de me proposer une stratégie d'épanouissement de la famille burkinabè et de promotion d'une responsabilité collective et de solidarité nationale à l'égard des plus démunis ». Je devais donc revenir quelques jours après pour lui soumettre mes propositions stratégiques. II a fait de même pour tous les membres du gouvernement.

\section{C'était une grosse responsabilité...}

Oui, j'avais 34 ans [Rires]! Au conseil des ministres, nous avions entre 30 et maximum 40 ans. Thomas Sankara lui-même était jeune, 34 ans. J'étais plutôt remplie d'angoisse... Mais quand j'ai commencé à concevoir la politique sectorielle, ses objectifs spécifiques et la démarche pour sa mise en œuvre, j'ai compris que l'occasion m'était offerte de prendre en compte les critiques et les idées de changement social qui m'envahissaient quand je parcourais les contrées rurales comme chargée d'études sociologiques. Très rapidement, je me suis sentie motivée et très engagée pour participer à l'élan de transformations socio-économiques, malgré la menace permanente d'être nous-mêmes débarqués violemment par un coup d'État. 


\section{Hadrien Clouet : À la table du conseil des ministres, y avait-il d'autres personnes que vous connaissiez?}

Nous étions à peu près tous de la même promotion [de la même classe d'âge], on se connaissait pour la plupart. Sauf les militaires présents avec nous, que je ne connaissais pas vraiment, mais ils n'étaient que quatre. L'ensemble du gouvernement comptait une trentaine de membres, en comptant le président Sankara, mobilisés par une volonté collective d'opérer des changements profonds.

\section{Et qu'aviez-vous comme moyens et comme équipe dans ce ministère ?}

Je me sentais plutôt seule au sein d'une vieille institution. Le personnel était motivé par ses activités pratiques très nobles, certes, mais mues par l'esprit d'assistanat. À l'exception du secrétaire général et du directeur de cabinet que j'avais choisis et nommés, la plupart des acteurs du ministère ne comprenaient pas ou n'acceptaient pas le processus révolutionnaire en cours. Mais ils manifestaient plutôt de la sympathie pour ma bonne volonté. Pour m'aider à conduire des réflexions stratégiques et à élaborer des programmes prioritaires, j'ai créé ce que j'ai appelé une cellule d'appui au sein du cabinet. J'ai identifié au sein du ministère une poignée de cadres expérimentés et motivés pour être membres de cette cellule.

Côté financier, la création d'une Caisse de solidarité nationale sous la tutelle de mon département ministériel offrait un instrument de sensibilisation et de mobilisation des populations pour une responsabilité collective. En plus, je pouvais utiliser les fonds disponibles pour réaliser des actions de secours d'urgence, d'investissements, de formation et de réhabilitation des groupes sociaux les plus vulnérables : victimes de catastrophes naturelles, enfants vivant dans la rue, populations en situation de précarité et de handicap, etc.

Thomas Sankara sensibilisait lui-même les populations par ces paroles: "C'est notre dignité qui est en jeu ; face à ces personnes qui meurent de faim au Sahel ou ailleurs, victimes de la sécheresse, de la faim, de la dégradation de leurs terres devenues arides et improductives, voulez-vous que nous nous transformions en mendiants internationaux pour les nourrir et leur porter secours, alors que nous avons la capacité de le faire par nos propres ressources ? ». En 6 mois, la Caisse de solidarité a encaissé [l'équivalent de] plus d'un million d'euros, issus de contributions volontaires spontanées.

\section{À la recherche de nouvelles formes démocratiques}

\section{En quoi consistait cette politique d'Essor familial ?}

Pour le côté «Essor familial », je me suis fixé trois directions. Premièrement, il fallait travailler en vue de l'amélioration des conditions économiques des femmes des quartiers populaires urbains et ruraux; pour ce faire, nous avons décidé de transformer les centres sociaux qui servaient à des activités d'éducation socio-domestique (cuisine, confection, etc.) en centres d'apprentissage de métiers générateurs de revenus (transformations alimentaires, tissage), sur un fonds d'éducation socio-sanitaire. Deuxièmement, nous voulions réhabiliter le statut de la 
femme au sein de la famille. Les femmes burkinabè étaient (et sont toujours) en majorité victimes des traditions qui sapent leur liberté et leur dignité. En matière de mariage, de veuvage, d'héritage, grand nombre de femmes sont soumises à des pratiques que nous voulions combattre légalement. En collaboration avec I'Union des femmes du Burkina, nous avons entrepris d'élaborer le premier Code de la famille. À l'époque, dans la sous-région Afrique de l'Ouest, les ministères de la Justice utilisaient encore le Code napoléonien, la tradition ou la religion pour traiter les problèmes liés aux droits des femmes et des enfants. $\mathrm{Ce}$ nouveau Code de la famille a été finalisé après la révolution et il accorde des droits. Mentionnons par exemple l'héritage aux veuves et aux orphelins, l'interdiction du lévirat ${ }^{8}$, la restriction de la polygamie et de la dot, l'interdiction du mariage forcé et précoce, etc. Troisièmement, il fallait exécuter les mots d'ordre qui venaient directement du président Sankara. Dans sa volonté d'instaurer l'égalité hommes/femmes, il a décrété ce qu'il a appelé le « salaire vital » pour les femmes mariées non salariées. II s'agissait de couper environ 1/3 du salaire des hommes mariés pour reverser systématiquement à leurs épouses en compensation des travaux domestiques et économiques qu'elles assumaient gratuitement pour l'entretien de la famille. Malheureusement, la mise en application de cette mesure était difficile voire impossible. C'était le rôle de mon département d'analyser les conditions d'opérationnalisation de cette décision. Lorsque des gens sont polygames, comment partager le salaire entre deux épouses ? À l'exception de ceux des fonctionnaires, les salaires du secteur privé n'étaient pas informatisés, ni soumis à une décision gouvernementale. Quelles modalités utiliser pour le reversement du salaire vital aux épouses des fonctionnaires ? Autant de questions dont nous n'avions pas les réponses sur le plan technique et institutionnel.

Thomas Sankara avait l'intention de réduire les salaires des fonctionnaires, dont au moins $70 \%$ étaient des hommes à l'époque, qu'il considérait comme trop élevés par rapport au travail fourni et injustes en comparaison des revenus du travail de la majorité des agriculteurs et des artisans du secteur informel. Sur ce point également, j'ai demandé qu'il accorde à mon équipe un temps pour étudier les implications socio-économiques d'une telle mesure sur les ménages concernés. Après l'accord du président, nous avons lancé sous ma direction une étude des revenus d'un échantillon de ménages urbains et péri-urbains dont les chefs de ménage étaient des fonctionnaires appartenant aux différentes catégories de la fonction publique. Les résultats de l'étude ont démontré que même les ménages les plus modestes vivaient ou survivaient avec des ressources nettement supérieures au salaire mensuel moyen de 50000 Francs CFA [90 euros]. Les ressources complémentaires étaient apportées à plus de $50 \%$ par des membres actifs du ménage, autres que le chef de famille salarié, notamment par les femmes et les filles : celles-ci fournissent chaque jour les combustibles, l'eau et des aliments issus de leurs petits commerces. Réduire les salaires aurait contribué à alourdir davantage la responsabilité des femmes. La solution devait être recherchée dans la valorisation économique des charges domestiques des femmes, mais surtout dans des investissements visant à approvisionner les ménages en eau et en énergie. Je crois que si je n'étais pas sociologue, je n'aurais pas tenté d'analyser le problème sous cet angle.

\footnotetext{
${ }^{8}$ Obligation pour une veuve d'épouser le frère du mari défunt.
} 


\section{Comment se jouait dès lors l'implication populaire, quelles formes de démocratie étaient en vigueur?}

J'ai été marquée et influencée par le mode de gouvernance démocratique du président Thomas Sankara. Il concevait la démocratie comme un processus de participation de toutes les couches sociales au développement national en forgeant un esprit de responsabilité collective. Cette démarche supposait que les populations soient informées sur les orientations de la politique révolutionnaire et qu'elles participent à un certain niveau de débats. Informer, sensibiliser et dialoguer étaient les principes de la gouvernance démocratique du président Sankara qui prônait la transparence et la reddition de la part de ceux et celles désignés pour conduire les rênes du pays.

Le système démocratique légué à nos dirigeants par la France depuis l'indépendance de la Haute-Volta reposait essentiellement sur l'organisation périodique des élections à suffrage universel du président de la République, des députés et des maires. Le système d'élection au suffrage universel était en fait la seule formule et le seul indicateur de démocratie que l'on nous a imposé depuis l'indépendance. Dès lors que le président de la République, les parlementaires et les maires de communes étaient ainsi élus, périodiquement, le pays était qualifié de démocratique par la communauté internationale. Mais quel est le niveau de compréhension des textes constitutionnels par une population à majorité illettrée ? Des textes sur la base desquels sont élus la plupart de nos dirigeants? Les meetings électoraux, tenus généralement en français et sans débats ni dialogues au niveau le plus local, ne suffisent pas à offrir aux populations une liberté de critiques, de propositions, encore moins de choix. Quel est le sens démocratique d'un tel système soi-disant démocratique?

En 1983, Thomas Sankara voulait changer tout ça, en jetant les bases de concertations systématiques et d'implication de la population. Lorsqu'il lançait un mot d'ordre ou une nouvelle mesure à portée nationale (par exemple "produisons et consommons burkinabè », «interdiction des feux de brousse et de la divagation des animaux afin de préserver l'environnement», " un village un bosquet pour encourager les plantations d'arbres sur l'étendue du territoire national », etc.), chaque ministre était chargé d'organiser «à la base » des meetings d'explication et de débats en langue locale, sur ladite mesure, avec les catégories sociales concernées par sa politique sectorielle. Avant ou après la publication d'une nouvelle, il nous conseillait, en tant que ministres, d'aller " chacun à sa base » (commerçants, artisans, femmes, agriculteurs, éleveurs, etc.) pour en parler et répondre aux préoccupations des populations. Thomas Sankara nous disait : «Allez sur le terrain, informez-les du mot d'ordre. Je veux que vous parliez dans leur langue, vous expliquez ». Vous voyez l'exercice là [Rires]! Moi, j'ai appris pas mal de concepts en français, et je ne les connaissais pas dans ma langue maternelle, par exemple la démocratie. Bref, il fallait débattre, laisser réagir et ainsi de suite, ce que je trouvais intéressant. Par cet exercice, Thomas Sankara voulait libérer la parole des gens, notamment celle des femmes et des jeunes. À l'occasion de toute réunion publique orchestrée par des services techniques ou politiques, une déléguée des femmes et des jeunes de la localité devait prendre la parole pour réagir.

Une autre formule de concertation démocratique était l'instauration des premières journées paysannes: il réunissait de 2000 à 3000 délégués paysans issus de toutes les 
provinces, sous de grandes tentes dotées de systèmes d'interprétation simultanée. Après avoir expliqué le sens d'une nouvelle mesure politique, il animait la discussion avec l'assemblée présente afin de comprendre leurs préoccupations et essayer d'obtenir leur adhésion. La réunion se tenait dans les langues locales grâce à l'interprétation simultanée. Les paysans se sont vite appropriés l'utilisation des micros individuels et du système d'interprétation. L'information et le débat sur des sujets politiques ou sociaux n'étaient plus réservés à la catégorie restreinte des personnes instruites et des experts travaillant dans l'administration, le secteur privé et les ONG. Thomas Sankara voulait démocratiser la prise de parole et l'accès à l'information et au savoir.

\section{Maxime Quijoux : Cela représentait une nouveauté ? Une rupture dans le rapport entre les élites et le peuple, par rapport à d'habitude ?}

Oui! Les débats libres entre la classe politique et des paysans constituaient une grande première. Après la révolution, le régime en place a continué à tenir des journées paysannes, des journées de la jeunesse, etc., mais il ne s'agissait pas de discussions, mais de questionsréponses entre les membres du gouvernement et des représentants de paysans ou de jeunes, choisis à l'avance pour exprimer des problèmes et poser des questions préalablement sélectionnées.

\section{Hadrien Clouet : Gardez-vous le souvenir de certains échecs dans votre ministère ?}

Un aspect sur lequel j'ai essayé de contribuer, sans y parvenir, concerne la mise en place des cités urbaines. L'amélioration de l'habitat urbain et l'accès de la classe moyenne au logement décent étaient une des priorités de la révolution. Des cités ont été bâties en lieu et place de quartiers urbains insalubres qui ont été rasés. Dans ces cités logeaient des fonctionnaires et des salariés du secteur privé, qui en réalité étaient une classe sociale homogène composée de ménages à composition restreinte. Ces logements n'étaient pas accessibles aux familles d'ouvriers, d'artisans, d'agriculteurs urbains, ni de travailleurs du secteur informel. Les personnes âgées y étaient rares. J'ai vu à l'époque en France ce qui se passait dans les cités ouvrières ou les cités HLM de banlieue parisienne. Des amis y habitaient, j'ai été témoin de la crise des jeunes et des précarités économiques et sociales vécues par les chômeurs.

L'harmonie sociale et les systèmes de solidarité se nourrissent de la cohabitation, de la mixité culturelle et des interactions sociales et socioprofessionnelles entre les anciens, les jeunes, les riches et les pauvres. C'est ma vision d'une société équilibrée. La richesse, le confort et la sécurité professionnelle ne sont pas garants d'un équilibre social. Je n'ai pas réussi à convaincre mes collègues sur les répercussions sociales néfastes des cités. En tant que sociologue, lorsque vous êtes dans un gouvernement qui agit rapidement parce qu'il faut livrer des résultats concrets et visibles à court terme, la réflexion collective est souvent sacrifiée.

Je ne parlerais donc pas d'échec mais plutôt de limites de ma contribution à la politique sociale de l'époque. L'obstacle essentiel était lié au fait que les changements visés, tels que l'amélioration des conditions de vie et du statut des femmes et des enfants au sein de la famille burkinabè, l'intégration sociale et économique des groupes victimes de marginalisation et de stigmatisation (jeunes de la rue, personnes soumises à la mendicité ou à la prostitution, etc.), 
et la sécurisation économique des femmes, impliquaient de nombreux facteurs, dont le moins important était le financement : il fallait construire une vision dans la durée, mener des actions d'éducation et de sensibilisation, utiliser une méthode d'intervention souple, évolutive et participative, impliquer des leaders politiques, sociaux et culturels, former le personnel du département.

Maxime Quijoux : À l'époque, qu'est-ce que cela impliquait d'être une femme ministre dans ce gouvernement ? Des particularités ou des expériences à part, au quotidien ?

Dans l'ambiance révolutionnaire de l'époque, la considération que le président Sankara accordait ouvertement et politiquement aux femmes constituait un rempart contre les discriminations sexistes et les harcèlements machistes au sein des membres du gouvernement. Vous vous sentiez à l'aise pour les remettre à leur place dans ce type de régime!

Le président Sankara défendait les droits des femmes et prônait l'égalité hommes/femmes qui se heurtait bien sûr à des mentalités très rétrogrades de l'époque. II a nommé cinq femmes ministres et c'était une première : à part moi qui étais chargée d'un département traditionnellement confié à des femmes, les autres étaient respectivement chargées du budget, de l'environnement, de la santé et de la culture.

Hadrien Clouet : Est-ce que vous rencontriez régulièrement vos homologues dans d'autres pays qui s'occupaient de l'essor familial ou de ce type de politique, sur le continent africain ou ailleurs?

Sous la révolution, nous n'avions pas le temps d'aller dans les grandes conférences internationales des ministres chargés des Affaires sociales ou je-ne-sais-quoi d'autre. Quand vous êtes ministre, dans un régime classique, vous passez la moitié de votre temps à voyager pour participer à des missions et à des conférences statutaires ou des rencontres régionales ou internationales. Mais sous la révolution, nous étions pris par le chantier de construction nationale. Le président Sankara disait : " Jetons les bases. Après, on aura le temps de souffler et de porter notre révolution à l'extérieur ». Cependant, le Burkina révolutionnaire a participé à la troisième conférence des Nations Unies pour les droits des femmes, tenue à Nairobi, en 1985. Notre pays était représenté par la présidente de I'Union des femmes du Burkina. 


\title{
Parcours de Joséphine Ouédraogo
}

- 1949 : naissance en 1949 à Koudougou (Haute-Volta)

- 1968 : baccalauréat à Paris (France)

- 1971 : diplômée de l'Institut de service social et de recherches sociales de Montrouge (France).

- 1974 : licence de sociologie à Paris V - René Descartes (France).

- 1974: chargée d'études sociologiques à la Société africaine d'études et de développement SA (Haute-Volta).

- 1980 : chargée d'études sociologiques à l'Autorité des aménagements des vallées des Volta (Haute-Volta).

- 1984 : ministre de l'Essor familial et de la Solidarité nationale (Burkina Faso).

- 1987 : Séjour à l'extérieur du Burkina. Consultante et coordinatrice de projets à I'Institut panafricain pour le développement à Douala (Cameroun).

- 1992 : chargée d'études et de la formation à la SACED à Bussigny (Suisse).

- 1993 : fondatrice et directrice d'ARC, bureau d'études S.A.R.L. à Ouagadougou (Burkina Faso).

- 1995 : directrice générale de la Coopération internationale au ministère des Affaires étrangères (Burkina Faso).

- 1997 : directrice du Centre africain pour le genre et le développement (CAGED) de la Commission économique des Nations Unies pour l'Afrique (CEA) à Addis-Abeba (Éthiopie).

- 2005 : secrétaire exécutive adjointe ad interim de la Commission économique des Nations Unies pour l'Afrique (CEA)à Addis-Abeba (Éthiopie).

- 2007 : secrétaire exécutive de l'ONG Enda-Tiers Monde à Dakar (Sénégal).

- 2013 : directrice d'ARC, bureau d'études à Ouagadougou (Burkina Faso).

- 2014-2015 : ministre de la Justice, des Droits humains et de la Promotion civique, Garde des Sceaux sous la transition (Burkina Faso).

- 2016 : ambassadrice du Burkina Faso en Italie et auprès des agences onusiennes de la FAO, du PAM et de la FIDAO basées à Rome (Italie).

\author{
Hadrien Clouet \\ CERTOP, Toulouse (France) \\ Maxime Quijoux \\ LISE, Centre National des Arts et Métiers (France)
}

\section{Bibliographie}

BIANCHINI Pascal (2002), «Entre instrumentalisation et autonomisation. Journalistes et militants dans les luttes scolaires et universitaires au Sénégal et au Burkina Faso (années soixante-quatre-vingt-dix) », Cahiers de la recherche sur l'éducation et les savoirs, 2002, 1, pp. 151-178. 
DABO Aïssata (2017), L'égalité de l'homme et de la femme dans le mariage en Afrique noire francophone. Étude comparée des droits du Bénin, du Burkina Faso et du Mali, Thèse de doctorat en droit privé, Université de Bordeaux et Université d'Abomey-Calavi.

Hagberg Sten, Kibora Ludovic, Ouattara Fatoumata et KonKobo Adjara (2015), "Au cœur de la révolution burkinabè », Anthropologie et développement, 42-43, pp. 199-216.

OUEDRAOGO Joséphine (1989) Rapport entre droit foncier traditionnel et droit moderne illustré par l'impact du régime Société sur le statut des femmes au Burkina Faso, Giuffré édition.

(1996), « Les femmes chefs de ménage en zone rurale du Burkina Faso », in J. Bisillat, Femmes du Sud, chefs de famille, Paris, Karthala, pp. 99-107.

Phelan Craig (2016), «Plus ça change: Trade Unions, the Military and Politics in Burkina Faso, 1966 and 2014 », Labor History, 57(1), pp. 107-125. 